\title{
Personalized and Diverse Task Composition in Crowd sourcing
}

\author{
M.Sowjanya, N.Ajay, A. Manikanta, G.Sudhagar
}

\begin{abstract}
We have a look at undertaking introduction in publicly supporting and the impact of personalization and decent variety on execution. A focal approach in publicly helping is undertaking, the element thru which employees find out undertakings. On mainstream ranges, for example, AMT, challenge is recommended via the functionality to kind undertakings by means of advent date or praise sum. Errand piece improves task by way of turning in for each laborer, a custom designed outline of assignments, alluded to as a Composite undertaking. CTs permit human beings to rapidly discover errands of intrigue. We propose diverse strategies for growing CTs and detail a streamlining problem that finds for a laborer, the most pertinent and differing CTs. We show that employees' experience is incredibly superior because of personalization that upholds an commercial condition of CTs with human beings' aptitudes and tendencies. We moreover check special techniques for broadening assignments in each CT. Assignment exceptional variety is grounded in affiliation thinks approximately which have indicated its impact on laborer concept. Our trials show that even as CTs enhance task throughput while contrasted with positioned facts, expanding errands provides to enhancing quit result first-rate. All of the extra explicitly, we show that errand throughput is super even as CTs incorporate undertakings having comparative subjects, whilst requester-based definitely respectable variety advantages every laborer upkeep and crowdwork first-rate. More

Specifically, we display that on the identical time as challenge throughput and expert preservation are awesome with positioned statistics, crowdwork extremely good arrives at its best with CTS differentiated by manner of requesters
\end{abstract}

Keywords-Throughput, Crowdsourcing, Optimization, urban zones, Clustering calculations, houses, publicly supporting, human variables

\section{INTRODUCTION}

Publicly assisting notoriety is commencing in one-of-atype areas as extra occupations appear to be "affirmed" and finished autonomously with the aid of masses of people. Miniaturized scale errands flow from honest demands, for example, decoding a shop receipt, to ones requiring the investment of proficient worker's, for instance, content material interpretation. A focal system in publicly helping is challenge, the device through which employees find out assignments. In this paper, we have a look at the hassle of

Revised Manuscript Received on September 14, 2019.

M.Sowjanya, Assist. Professor, Department of CSE, Siddhartha Institute of Technology \& Sciences, Narapally, Ghatkesar, Hyderabad, Telangana, India.

N.Ajay, Assist. Professor, Department of CSE, Siddhartha Institute of Technology \& Sciences, Narapally, Ghatkesar, Hyderabad, Telangana, India.

A. Manikanta, Assist. Professor, Department of CSE, Siddhartha Institute of Technology \& Sciences, Narapally, Ghatkesar, Hyderabad, Telangana, India.

G.Sudhagar, Professor, Department of ECE, Siddhartha Institute of Technology \& Sciences, Narapally, Ghatkesar, Hyderabad, Telangana, India turning in, for every laborer, a custom designed synopsis of undertakings and concentrate its impact on execution.

Publicly supporting has caught the eye of the arena as of late 1.

Therigorousengineeringdisciplineofsoftwaredevelopment,su chasrigidsyntax and semantics of programming dialects, displaying dialects, and documentation or procedure benchmarks.

The inventiveness additives of programming Requirement examination, shape, finding out, and evolution.Theissueistostimulateandpreservecreativityinthese softwaredevelopment assignments through aggregate information?

-The mind studies issues of publicly helping, for example, competition, motivator, acknowledgment, sharing, coordinated strive, and gaining knowledge of. The mind technological knowledge ought to be focused even as concurrently cordial, social succesful, getting to know, and individual fulfillment for individuals, requesters and directors.

The financial attitude so fall parties which includes requesters, publicly helping stages, and individuals.

fine views which incorporates aim tendencies, for instance, utilitarian accuracy, execution, safety, dependability, viability, wellbeing, and difficulty tie characteristics, for example, ease of use.

On this detail, we plan to altogether talk approximately and have a look at key requirements of programming publicly helping to inspire making plans, improvement and renovation of an collection of great scale programming frameworks. The the relaxation of the element is composed as pursues: Sect.2 defines the belief of "programming publicly assisting" and contrasts it and different favored programming development techniques which consist of spry programming development, re-appropriating and open supply. Section3 depicts Crowdsourcing for large-Scale software application development 5 the standards of programming publicly supporting which consist of codevelopment, centered development and offense-barrier based definitely quality confirmation. Section 4 offers a Cloud-

basedsoftwarecrowdsourcingarchitectureforcrowdworkforce coordination and management. Section5 offers a four-level improvement model for the evaluation programming publicly supporting manner from the additives of framework's scale, challenge time stages, designers and programming levels. 


\section{WRITING SURVEY \& METHODOLOGY}

A Survey Of general-cause Crowdsourcing techniques Anand Inasu Chittilappilly, Lei Chen, Sihem Amer-Yahia

In view that Jeff Howe supplied the time period Crowd sourcing in 2006, this human-managed critical questioning worldview has accelerated a splendid deal of interest and has been a hot research subject inside the challenge of software engineering. Notwithstanding the fact that a top notch deal of hard work has been directed on this trouble, to date we do no longer have a much accomplishing have a study on maximum vast art work finished within the publicly helping subject. In this paper, we plan to provide a state-of-the-art image of the prevailing decreasing element systems thru and massive cause publicly supporting. As in keeping with their center, we isolate this art work into 3 sections, which can be: motivating force configuration, project, and pleasant manipulate. For each thing, we start with diverse troubles looked around there pursued with the useful resource of a concise portrayal of modern artwork and a discourse of upsides and disadvantages. Furthermore, we furthermore gift a true situation on how the diverse strategies are implemented in executing a place primarily based publicly supporting degree, critical. At long closing, we function the restrictions of the triumphing extensively useful publicly assisting techniques and present a few open problems right here.

Gadget design For Crowdsourcing Markets With Heterogeneous duties

\section{G. Goel, A. Nikzad, and A. Singla.}

Structuring perfect estimating arrangements and additives for assigning errands to employees is essential to internet primarily based completely publicly assisting markets. On this paper, we don't forget the accompanying practical putting of online publicly supporting markets. We are given quite some heterogeneous undertakings requiring sure competencies; every laborer has sure aptitude and pursuits which represent the arrangement of errands she is eager on and geared up to do. Given this bipartite chart among people and assignments, we plan our tool TM-UNIFORM which does the allotment of errands to workers, at the equal time as making positive spending opportunity, motivating strain similarity and accomplishes near ideal software. We similarly growth our consequences by way of misusing a reference to online advert terms distribution trouble and present a randomized tool TM-RANDOMIZED with superior bet guarantees. Apart from solid hypothetical ensures, we do substantial experimentation making use of reenactments truely as on a practical contextual investigation of Wikipedia interpretation mission with Mechanical Turk employees. Our outcomes show off the affordable appropriateness of our systems for sensible publicly helping markets at the net.

\section{OVERVIEW OF CROWDSOURCING BASED SOFTWARE DEVELOPMENT}

Modern high-quality and complex programming frameworks 3demonstrate the accompanying conspicuous attributes: extraordinarily decentralization of frameworks with heterogeneous and differing components, actually conflicting, mysterious, and various prerequisites simply as

ceaseless improvement and enterprise. Every this kind of highlights thoroughly negate the most significantly identified as summations made by way of the same old programming building philosophies. As programming development is especially iterative, programming improvement forms frequently intensify on conveyance of center of the road reviews, as an instance, necessity statistics, shape records, test plans, test data. Mainly, in plan-driven touchy product improvement techniques, as an example, Water fall version, Spiral model, and versiondriven technique, it is traditional that a meeting of programming designers can make unique research of companions' prerequisites and define a reliable and deliberate diagram for programming engineering and nitty gritty development intend to actualize every utilitarian necessity. In addition, all of the product improvement physical video games may be very an lousy lot planned and gain relentless advancement towards the predefined achievements that purpose absolutely attempted arrivals of the running programming frameworks. None of those approachs can bolster moderate-footed and bendy programming advancement of substantial scale programming frameworks due to regular modifications in client necessities and dynamic prepared conditions. To cope with those issues, programming development paperwork have developed from the conventional arrangement pushed manner to later mild-footed strategies four, circulated programming development 5 , open-source approach 6 , and community driven programming development. Those reducing component programming approach advocate three widespread additives to trade programming building systems.

(1) Adaptive software program software creation and Evolution: software program program designers need to have the choice to continuously improve and refresh programming frameworks to satisfy the dynamic of purchaser necessities.

(2) network and Social Intelligence: to overcome the unpredictability of widespread scale programming frameworks, programming ventures need to take advantage of aggregate records and difficult work of designer networks to have an on-request team of workers with diversified capabilities and gifts. $6 \mathrm{~W}$. Li et al.

(3) Open and allotted method for software program Innovation: An open and disseminated programming gadget is critical for encouraging network based absolutely programming development and development. Network people need to be very an lousy lot prepared to attend to trying out problems in programming ventures. Publicly assisting is an on the net, dispersed important wondering and advent model that use the combination notion of online networks for specific the executives goals. Certainly, it's far promising to use the rule of thumb of thumb of publicly supporting in the place of programming advancement and joins all the above fixings to empower a few different product worldview-programming publicly assisting.

What's software program Crowdsourcing? 
Programming publicly supporting is an open call for investment in any assignment of programming development, in conjunction with documentation, plan, coding and checking out. The ones undertakings are normallyconductedbyeithermembersofasoftwareenterpriseor peoplecontracted by using the mission. Be that as it could, in programming publicly helping, every one of the errands can be doled out to every body within the fundamental populace. Thusly, programming publicly helping no longer truly trap giant degree requirements and first-rate houses regarding community pursuits, but further encourage quit-clients to make their non-public tweaked programming administrations. Along those traces, programming publicly supporting is largely community primarily based totally programming development worldview.

Programming publicly assisting receives a bendy, open and circulated development manner. Rich commitment with both patron and engineer networks empower software program publicly assisting experts to rapidly react to new encourage backs and requests from their customers and make go to and in-time enhancements for his or her product. Internet primarily based definitely programming diploma with worked in on line network and marketplace functionalities encourages facilitate a huge wide variety of circulated community individuals in the improvement and development of massive scale programming frameworks. Understood programming publicly assisting degrees inclusive of Apple's App keep, TopCoder 7 display the fulfillment of programming publicly assisting processes as a long way as programming environment extension and object quality development.

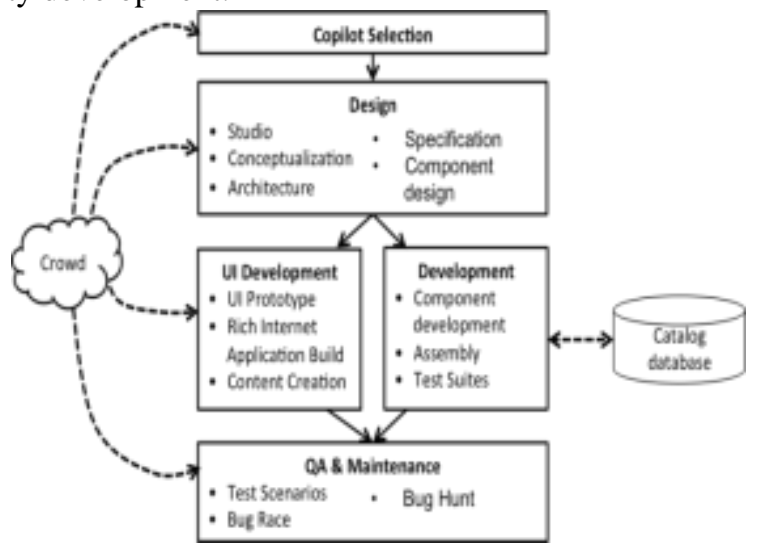

Fig:- Architecture Design

\section{ONLINE SOFTWARE MARKETPLACES FOR SOFTWARE CROWDSOURCING}

Apple's App save is essentially a web IOS utility business middle in which IOS dealers can straightforwardly convey their objects and imaginitive systems to PDA forestall customers. This flexible programming marketplace provided the micropayment and positioning device to empower planners to select out up blessings from utility downloads. The excessive positioning and down load pace of a seller's iPhone application receives considerable gain for him. This market-arranged motivator persuades sellers to contribute innovative plans to the economic center and to attract in greater downloads from clients of the App keep. Moreover, through manner of posting comments and surveys on the IOS programs available within the business center, customers can expressly introduce their conditions to application planners. There are focused connections among traders taking a shot at a comparable beauty of IOS programs. They want to unexpectedly react to the criticisms and increasing requests from their customers and decorate their product to tie down their favorable characteristic to big contenders and hold their clients. Each any such units of the App shop boost the exponential improvement inside the IOS surroundings. Inner under four years, Apple's App keep has become a enormous flexible software surroundings wherein as a good deal as a hundred and fifty,000 dynamic distributers have made more than 700,000 IOS applications. However the authentic IOS engineer application gave via Apple, severa IOS software architects despite the fact that want useful and easy channels to search for financing and software program application engineers for developing novel application thoughts, collecting greater consumer audits and trying out packages earlier than discharging them on the financial center.

In this manner, numerous community primarily based, community stages were rising as superior mobile phone applications hatcheries for the App save. As an instance, App keep (www.Appstori.Com)introduces a collection subsidizing manner to address fabricate an online network for developing promising mind concerning new iPhone packages. It offers a "overview" window for iPhone utility aficionados to pick their desired mind, bolster and efficaciously participate inside the promising ventures. Fashioners are urged to advocate assignment mind to the survey board who assesses the oddity of the project pointers and award them into the formal App save ventures. After their proposition receives endorsement, each App shop undertaking group gauges development exertion together with its cutoff time and spending plan, and connects with swarm for financing manual through the App keep degree.

Annoying situations And Tournaments For software program software Crowdsourcing

Each different publicly supporting version-top Coder, makes a product project version in which all the product improvement undertakings are composed as worrying situations and the engineer of the pleasant association wins the pinnacle prize. Considering that 2001, pinnacle Coder has constructed up enterprise on line level to help its biological machine and acquire a digital international team of workers with in extra of 250,000 enrolled humans and 50,000 dynamic people. Exam amongst software software Crowdsourcing and one of a kind software program improvement strategies

As every other product development strategy, programming publicly assisting acquires severa significant thoughts from exceptional contemporary programming development strategies which incorporates spry programming advancement, re-appropriating and open deliver. Essentially, programming publicly helping is an appropriated and open programming development worldview with the concept of online work marketplace and engineer community. As regarded in desk 1, the large

Blue Eyes Intelligence Engineering

\& Sciences Publication 
likenesses and contrasts among programming publicly supporting and one-of-a-kind strategies are abridged inside the additives of flow, coordination, transparency and improvement Incentive.

Spry software program improvement instead of software program Crowdsourcing

Spry programming improvement changed into proposed to address the dearth of plan-pushed methodologies with the presentation of iterative and constant improvement. With versatile arranging, transformative advancement, and an iterative coding approach, coordinated method can basically quicken the conveyance of recent programming highlights in light of the fast trade in purchaser prerequisite. Its affiliation stresses co-located up close to and personal connection, in which programming engineers and a purchaser delegate may have constant correspondence and communication through the entire lifecycle of programming improvement. Virtually, unadulterated spry techniques are maximum suitable for little predicted or medium-sized undertakings, and endeavors to scale them up for great and complex activities, typically bring about re-reception of often traditional practices [9]. As of now, maximum studies endeavors on the extremely good way to adopt a cross breed approach of plan-driven and dexterous method center around intraaffiliation situation, in which deft style improvement is grasped on the organization diploma and the conventional forthright arranging clearly as improvement device is obtained in the assignment level. Honestly, regardless of the half of and half of of technique, every one of the acts of dexterous techniques are often restricted within the restriction of companies and affiliation. Conversely, publicly assisting strategies bolsters large scale, disseminated on-line joint attempt for put it up for sale pushed colossal programming technology, defeating the

Table 1 Comparison between software crowdsourcing and other methods

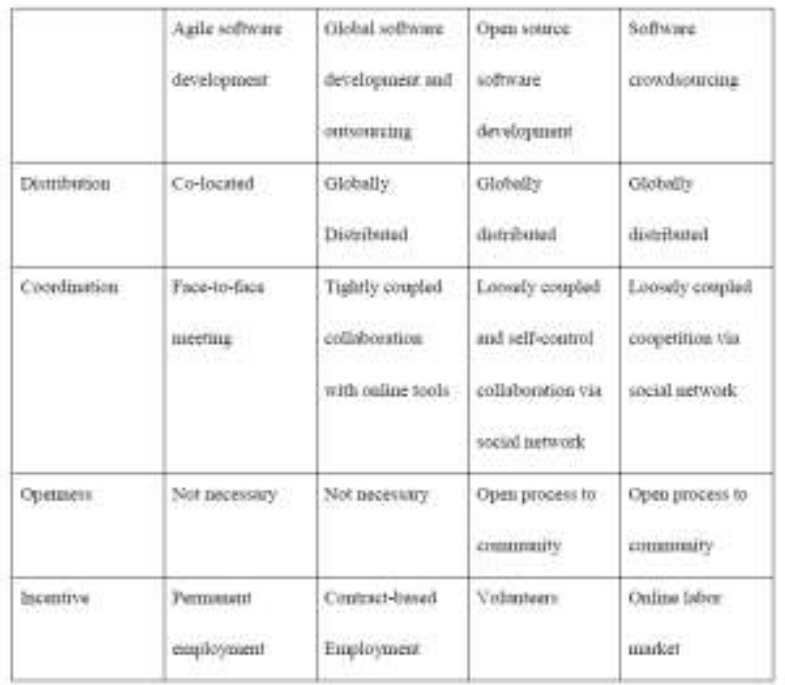
dexterous approach. The human beings institution organized management shape of publicly supporting empowers us to efficiently outfit the scholarly belongings for the development of large scale programming frameworks. All of the extra extensively, determined thru the praise and various consumer requirements, community people can act clearly
Obstacle of both association driven technique and

sorted out into severa nimble companies that would rapidly gather improvement undertakings and bring awesome items.

Requirements Of software program program Crowdsourcing For software application surroundings

Practices of programming publicly supporting may additionally take numerous methodologies to accomplish certainly one of a kind task desires, as an example, widening cooperation, searching out novel arrangements, distinguishing abilties and getting high-quality programming devices. In any case, they want to comply with comparable pointers which can be imperative to the development and thriving of a energetic programming surroundings. This region gives three everyday requirements of programming publicly helping along with Co-Innovation, aggressive software improvement and Offense-protection primarily based excellent warranty.

\section{CO-INNOVATION AND PEER-PRODUCTION}

Publicly supporting programming improvement underscores the essential guiding principle of Co-advent, Co-plan and Co-improvement, which empowers huge willpower of community individuals within the improvement of large scale framework organic structures. This guiding principle empowers every network detail together with prevent-customers, talented software program engineers and space professionals with dynamic jobs in help of programming improvement, which is going past the restrict of the normal programming development rehearses.

Associate production and Egalitarian design system

The importance of large virtually worth and the process of significant well well worth creation in contemporary programming development are brief shifting from a product item and fashion designer pushed view to customized patron encounters. Educated, prepared and dynamic consumers are step by step co-making an incentive with the expert designers. Companion generation of programming publicly assisting pursues a populist configuration approach, in which sizeable portions of ordinary stop-clients are enabled as co-makers or co-architects to attract in with a enormous range of resourceful carrying activities giant to programming creation, as an example, easy notion approval, coordinated effort on conditions, comments on ease of use, and in any event, programming and attempting out. With natural and easy to-employ programming gadgets, trendy surrender customers can't without a doubt function programming programs thru contrasting their exhibition and excellent, but additionally create mild-weight programs and tweak specific highlights to meet their various purposes and requests. Accomplice era cultivates and quickens the upward push of an open level programming natural device widening swarm help and associating degree sellers, programming designers and customer networks. The success of a huge scale programming publicly supporting mission basically is based on the size and movement of such an engineer network. Beyond investigations on open supply organic systems $[10,11]$ discover that most open deliver extends inside the full-size open source degrees, as an 
instance, supply manufacture and acquire actually have predetermined type of dynamic people. Finally, it's miles simple for a product publicly helping assignment to take all of the possible techniques together with budgetary help, scholarly asset of development and identifying how to pick whatever range participants as may be predicted below the times.

Innovativeness and variety in software program program Crowdsourcing businesses

Publicly supporting desires a various community of agency employees in disseminated regions with severa basis to guarantee a first rate fashion of assessments are communicated to empower innovativeness, and to keep away from anybody-sided of man or woman tasks interior a selected community. Especially even as the giant goal of a product publicly helping assignment is to research novel and top notch mind for destiny modern-day artwork, originators must be urged to endorse a extensive scope of thoughts and fashions for an open soliciting for.

This displays in reality certainly one of imagination standards low restrict, immoderate roof and vast dividers [12]. Programming publicly assisting often sets up a low limit to induce more individuals to take an assortment of programming development undertakings to growth the network. It likewise gets immoderate roof method to display qualified developers for mind boggling and testing ventures. As constant with the usual of large divider, stages and units of programming publicly supporting must introduce sufficient opportunity for participants to investigate new measurements and look for creative arrangements

\section{CONTENTION IN SOFTWARE CROWDSOURCING}

The technique of programming publicly helping frequently suggests strong focused features, which takes after the technique of paper strength of mind in a outstanding meeting. The dedication of a product publicly assisting mission along with the specialized prerequisite, time critical, and prizes for the paintings, is publically noted to the network as an open require the satisfactory association with the aid of opposition.

As a huge response to this name, network individuals, who are eager in this venture, can start to take on the task and gift their outcomes to an audit leading enterprise of the community,

Who's responsible for assessing the individual of their quantities and pick a final conclusion for the closing champs.

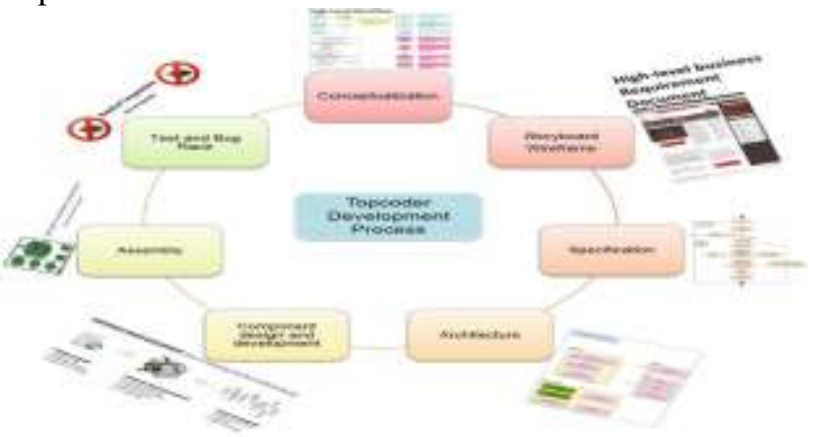

Fig. 1 TopCoder competitive development process

- undertaking Solicitation:

A TopCoder venture leader characterizes the prerequisite in not unusual language and distributes the element of an mission at the degree to require the determination of any capability engineers in the community. Every now and then he can determine the functionality requirements of the errand to allow sincerely the engineers with exquisite aptitudes and skill to enlist.

-Answer Bidding:

Designers with the right statistics base and ranges of competencies for the project check within the TopCoder project and begin to take a shot at the errand depending on their comprehension of the necessity. On the off chance that they finish the errand within the time hassle, they're able to present their solutions for the survey corporation of the assignment as presenting for the victor prize.

-evaluation and profitable:

The survey group assesses every one of the entries and springs to a decision victors out of the terrific entries. Just the entries with as well as may be expected probable win the prize ultimately. What's extra, the selected affiliation and audit proposals can be applied as transitional substances inside the following degree of the mission. Truely, such an aggressive approach dreams seeking out the awesome association with the highest caliber from numerous yields for the equal improvement errands. However the inducement at the back of

First-class screening, it likewise figures out a way to lessen the fee of hard work by using the usage of just paying the champs amongst each one of the individuals. Be that as it can, this fee decrease has negative impact on nontriumphing organizations thinking about that their endeavors and commitments won't get any economic cease result. Along the ones lines, feeble companies may lose their inspirations to encompass the venture inside the event that they have from time to time any possibility to outflank their opponents. To deal with this problem, non-monetary prizes need to be available in the assignment as strengthening motivators for the members.

For instance, a mission run by means of a well-known IT employer may have numerous contenders who are keen to partake to enhance their notoriety positioning irrespective of whether the corporation may additionally moreover provide moderately low victor prize. The non-coins associated motivation desires to artwork on the subject of synergistic network wherein individuals esteem notoriety, learning possibility, profession improvement and philanthropy

\section{JOINT EFFORT IN SOFTWARE CROWDSOURCING}

As joint effort and coordination are the essential components in any dispersed programming method, all of the product publicly assisting degrees bolster pretty lots cooperation among their takes an hobby. For example,

severa on line IOS people organization, as an instance, App save (www.Appstori.Com) and iBetaTest (www.IBetaTest.Com) deliver communitarian programming 
publicly assisting scenes to connect iPhone software originators, IOS engineers, beta-analyzers, financing individuals and regular customers together. Therefore, little organizations or people on iPhone utility advancement can search for outside subsidizing, get help to run beta-testing on early types of their applications, and gather essential consumer criticisms in advance than discharging their devices onto the notably targeted App shop. Determine 2 shows the widespread players in the foundation of AppStore:

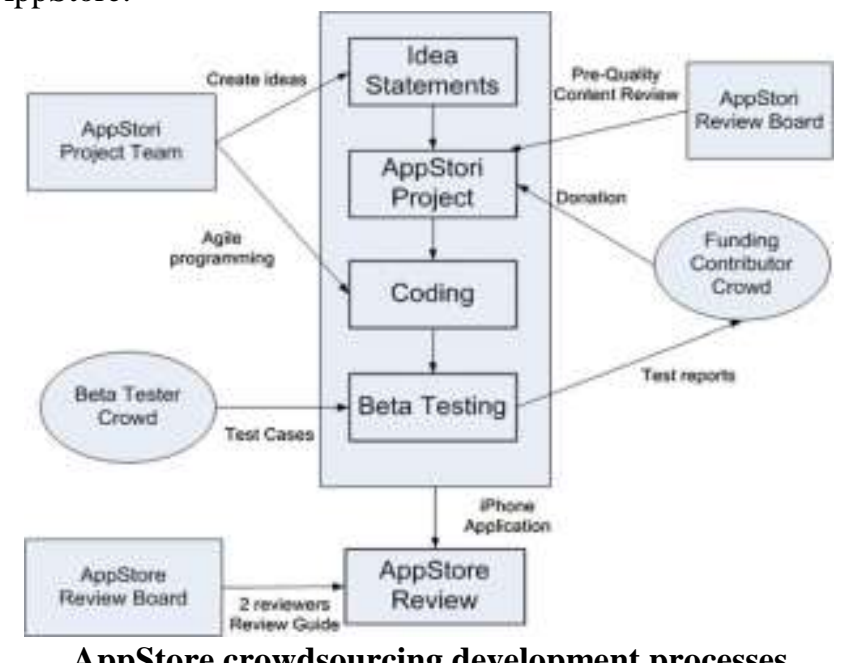

AppStore crowdsourcing development processes

- Crowd funding and Stakeholders:

Like Kickstarter.Com within the coins related market, any AppStore thing can post an original concept of IOS software onto the AppStore, identifying the venture goals and cutoff times, and request financing. After the AppStore evaluation Board favors the project, any individuals who love the possibility of the venture can change into a companion thru financing the undertaking. Albeit a associate can not pull again its store in advance than the finish of the mission, the person can test the advancement of the enlarge and speak to for the challenge leader to finish the task indoors a predetermined time allotment. Some thing else, the financing won't be moved to the engineer institution towards the quit.

-Transparency and Agile improvement:

AppStore empowers a hundred\% straightforwardness the various dressmaker company and the network. Every colleague need to supply nitty gritty individual profile to introduce their enjoy and task within the venture. What is more, the venture repute want to be refreshed so as to reveal the improvement to the network. Any man or woman who is happy to be a piece of the organisation can make a contribution thoughts to direct the undertakings improvement manner, supply social assist to the undertaking through way of internet-based totally totally social networking development, or maybe emerge as beta analyzers for fine confirmation. The beta analyzers can determine early styles of the software program under take a look at and deliver direct inputs and malicious program reviews to the designers.

-Stimulating Creativity in cell software improvement: AppStore empowers studies exceptional approaches and grasping wealthy patterns to make progressive transportable packages. Net 2.0 correspondences make it less tough for

benefactors, engineers, and analyzers to change mind, publish feedback and make revel in of extraordinary thoughts. Software shops open and innovative circumstance empowers numerous cycles of experimentation closer to the fruitful shape until it thoroughly may be prepared for distribution on App save.

-understanding sharing and reading:

AppStore is an splendid spot for human beings to adapt new applications, innovations, and pattern in transportable packages, and flexible customers and devotees can associate legitimately with portable engineers and business visionaries for direct inputs. The ones criticisms are expensive to accumulate previously.

\section{PROGRAMMING CROWDSOURCING SHAPE AND FASHIONS}

Due to diverse variety and separation the diverse individuals of programming publicly assisting obligations, it is fundamental to have a Cloud-primarily based programming level to help big scale open and conveyed development paperwork in programming publicly assisting. Envision an huge horde of engineers positioned in diverse time zones attempt to cooperate on a similar programming task. A cloud furnishes them with a flexible level with precise enough property, which encompass processing strain and programming databases. With the rising cloud programming gadgets, as an instance, DevOps (a portmanteau of improvement and sports) [14] and massive scale programming mining, it altogether decreases the diploma of physical paintings required in installing region programming advent situations and engages peer engineers to perform programming publicly helping assignments productively in configuration, coding, and testing.

\section{PROGRAMMING CROWDSOURCING SHAPE}

But various desires and systems acquired with the useful resource of programming publicly assisting strategies, they truly percent loads of shared trait in time period of stage assist. As an internet paintings exhibit where swarm workforce can independently select an collection of programming improvement undertakings noted thru task initiators, a product publicly helping degree wants to effectively encourage collaboration among mists $\mathrm{C}$ human cloud and machine cloud. Many middle administrations concerning the artwork the executives and project management need to be joined into the degree together with expertise positioning, institution arranging, undertaking coordinating, and compensating in reality as enterprise financing. Similarly, every character have to have the choice to successfully instate a virtual workspace with plan and coding programming units altered for explicit errands in a publicly supporting challenge. Maximum of those number one components are typified in reference engineering of cloud-based totally definitely programming publicly

assisting framework, regarded in Fig. 3. There are three considerable gatherings of programming units in a cloudbased totally programming publicly assisting: 


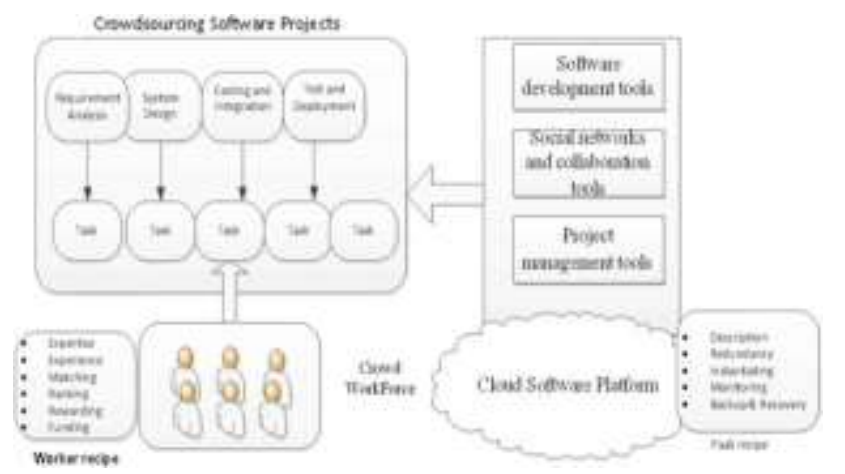

Reference architecture for cloud-based software crowdsourcing

\section{SOFTWARE DEVELOPMENT TOOLS\& RESULTS}

In any product publicly helping obligations, swarm group of workers goals demonstrating apparatuses, reenactment units, programming language devices, as an instance, compilers and astute editors, plan documentations, and trying out gadgets. An IDE for publicly assisting can incorporate those apparatuses for necessities, plan, coding, compilers, debuggers, execution exam, attempting out, and preservation. As an example, cloud programming layout gadgets, as an instance, Chef (www.Opscode.Com/cook dinner/) and Puppet (puppetlabs.Com) allow network humans to build up their very non-public virtualized development situation. Guide lessen based totally log the board apparatuses bolster huge scale framework log organisation and studies, and empower community people to determine programming problems and enhance framework unwavering splendid utilising log messages.

- Social community and Collaboration tools:

$\mathrm{Fb}$, twitters, wikis, net journals and specific webprimarily based coordinated strive gadgets allow individuals to impart for sharing and joint effort. For example, facebook profiles can be upgraded to encourage the association of a virtual publicly helping organization regardless of whether the participants do now not beforehand have any acquaintance with one another. A shared chalkboard based level can be implemented in which people can see a normal location and suggest mind to enhance the preparations published there. Novel money related gadgets for cloud installment and digital credit rating the executives can bolster task subsidizing deliver and economic trade between project initiators and group personnel.

- venture management tools:

Publicly supporting task the board want to bolster undertaking fee estimation, advancement arranging, primary leadership, computer virus following, and programming archive protection, all precise for the setting of the dynamic designer community. But these ordinary capacities, it desires to enroll in publicly helping specific administrations, as an example, positioning, notoriety, and deliver frameworks for the 2 items and people. For example, TopCoder gives a complicated positioning plan, like recreation competitions, to rank the aptitudes of community people in programming development. Network human beings frequently determine to take an interest in a particular undertaking within the event that they recognize the website of the individuals formerly selected. Via DevOps advances, Cloud ranges permit customers to depict their personal product stacks in form of plans and cookbooks. With the appropriately decided PaaS plans, programming publicly helping undertaking chiefs can set up a redid cloud programming situation to encourage programming

Publicly helping technique:

(1) a high makes use of programming structures administration and cooperation devices to configuration remunerating thing to persuade swarm people and undertaking the executives gadgets to prepare advancement assignments amongst swarm body of workers thru positioning mastery of everybody and coordinating their aptitudes with the numerous tiers of undertakings.

(2)in the direction of a specific programming undertaking, director sets up a digital framework degree with all of the vital programming development riggings to help swarm people with their publicly assisting assignments.

(three) each cloud degree and paintings technique can paintings in a versatile way to make fee-effective asset usage. Contingent upon the estimation of programming item and size of programming advancement, a mission director can suggest the best spending plan to drag in something quantity ability designers as may be allowed and association figuring property to help the development physical sports.

\section{Algorithmic answer}

Our manner to cope with creating CTs expands on a fluffy grouping calculation like k-implies. We first present the vital circle of the calculation, that iteratively refreshes centroid positions and CTs till intermingling. At that component we center across the potential that constructs a $\mathrm{CT}$ around a centroid, and gift our keen manner to deal with tackling this problem.

\section{A. Convergence circle}

The issue of building composite undertakings has likenesses with building agent composite matters. Therefore, our calculation for building CTs broadens the KFC calculation [6] and receives the shape of fluffy bunching, as depicted in set of policies 1. Fluffy bunching begins offevolved by way of using introducing $\mathrm{C}$, the affiliation of okay centroids to arbitrary positions (line 2). W is the masses' framework speaking to the cooperation of every errand from $\mathrm{X}$ to every company. Fluffy grouping iteratively refreshes masses (line 6) and centroid positions (line eight) till aggregate (line nine) [14]. So additionally to KFC, at some thing component centroids are refreshed, a CT is artwork around every centroid (line 7) as a manner to refresh $\mathrm{Su}$, the arrangement of adequate composite undertakings worked for the laborer u. To choose the assignments added to every $\mathrm{CT}$, we depend on a choice capacity f, it's nitty gritty in segment III-B. Parameter $\alpha, \beta$ and $\gamma$ are step by step modified in accordance with their goal functions over $\eta$ cycles (traces 4 and 10). Consequently, the calculation starts offevolved offevolved with the resource of executing a desired fluffy bunching ( $\alpha=1, \beta=\gamma=$ zero), which guarantees that centroids are all round positioned,

earlier than grade by grade expanding the importance of cohesiveness personalization in resulting cycles (line 5). 
The replace rules for hundreds and centroids whilst using cosine similitude are surrendered at the same time as our mission definition varies from the only of KFC, the laborer personalization time period does no longer encompass the scenario of the centroids, so the update regulations are the equivalent. Nevertheless, the ability that fabricates the CT concerning a centroid, spoke to in algorithm 1 by the use of $\mathrm{f}$, should be adjusted to represent the specificities of our difficulty. We painting that adjustment subsequent.

\section{DESTINY ENHANCEMENT}

The publicly helping sports are defined by way of the dimensions of improvement agencies, the scale of programming frameworks a work in progress, the challenge term and tiers guide for programming advancement bureaucracy. Programming publicly helping is a organized and promising vicinity for check out which includes demonstrating, exam, replica, experimentation, and bolster condition development. There are numerous future studies headings such as hypothetical fashions, streamlining techniques, foundation bolster highlights, and social problems. Hypothetical devices, as an instance, undertaking speculation and monetary models want to be implemented to depict cooperative electricity amongst singular endeavors, motivator additives, and object high-quality and project imperatives. Formalized programming publicly helping system fashions and enhancement strategies should be created to meet the correct goals of programming publicly helping undertakings in destiny.

\section{Give up}

Cloud-primarily based totally programming publicly supporting is every other approach for minimum attempt brief programming advancement, and the presence of great biological structures has indicated that this method is viable. This element offers the because of this of programming publicly helping and completely examines each the precious sizeable and calculated models of this growing approach. As an open name for investment in any undertaking of programming improvement, programming publicly helping may be looked after out in diverse manners, as an example, online programming market, on-request work asset and communitarian networks. Essentially, programming publicly assisting is an appropriated and open programming development worldview that acquires numerous significant mind from extraordinary contemporary programming development techniques along with spry programming improvement, re-appropriating and open source. In any case, the awesome highlights of programming publicly helping which encompass inexactly coupled coordination, open method and marketplace-pushed motivating pressure machine, make it practicable to address with the troubles of giant scale programming development. In this section, we define 3 normal requirements of programming publicly assisting together with Co-Innovation, competitive software application development and Offense-protection based totally first rate warranty. To pursue the standards and bolster full-size scale programming development with swarm, a product publicly supporting degree desires to encompass a cloud based totally totally programming engineering and fuse center administrations for successfully encouraging cooperative electricity among mists $\mathrm{C}$ human cloud and gadget cloud. Furthermore, we gift a four-degree improvement model because the cause for looking at and surveying programming publicly supporting method..

\section{REFERENCES}

1. P. Mavridis, D. Net Amblard, and Z. Miklo's, "making use of numerous leveled talents for upgraded task in facts escalated publicly assisting," in courtroom cases of the 25th global convention on worldwide huge internet, WWW 2016, Montreal, Canada, April 11 - 15, 2016 , 2016, pp. 843-853.

2. S. B. Roy, I. Lykourentzou, S. Thirumuruganathan, S. Amer-Yahia, and G. Das, "assignment mission improvement in statistics escalated publicly supporting," VLDB J., vol. 24, no. Four, pp. 467-491, 2015.

3. S. Amer-Yahia, F. Bonchi, C. Castillo, E. Feuerstein, I. Me'ndez-D'1az, and P. Zabala, "Composite restoration of various and important corporations," IEEE Trans. Knowl. Information Eng., vol. 26, no. 11, pp. 2662-2675, 2014.

4. H. Bota, k. Zhou, J. M. Jose, and M. Lalmas, "Composite healing of heterogeneous net seek," in twenty 0.33 worldwide world considerable net convention, WWW '14, Seoul, Republic of Korea, April 7-11, 2014, 2014, pp. 119-130.

5. A. Brodsky, S. M. Henshaw, and J. Shave, "Card: a preference direction shape and application for suggesting composite different options," in RecSys, 2008, pp. 171178.

6. V. Leroy, S. Amer-Yahia, E' . Gaussier, and S. H. Mirisaee, "building agent composite matters," in complaints of the 24th ACM Inter-countrywide on conference on statistics and knowledge control, CIKM 2015, Melbourne, VIC, Australia, October 19 - 23, 2015 2015, pp. 1421-1430.

7. A. Holy messenger, S. Chaudhuri, G. Das, and N Koudas, "Positioning articles dependent on connections and stuck dating," in EDBT, 2009, pp. 910-921.

8. S. B. Roy, S. Amer-Yahia, A. Chawla, G. Das, and C $\mathrm{Yu}$, "building and investigating composite things," in SIGMOD conference, 2010, pp. 843-854.

9. M. D. Choudhury, M. Feldman, S. Amer-Yahia, N. Golbandi, R. Lempel, and C. Yu, "Programmed improvement of motion schedules utilizing social breadcrumbs," in HT, 2010, pp. 35-forty 4.

10. A. Graham, H. Garcia-Molina, A. Paepcke, and T Winograd, "Time as pith for picture br 